\title{
From regular to irregular solid electrode surfaces: a systematic approach to their characterization
}

\author{
A.J. Arvia *, R.C. Salvarezza \\ Instituto de Investigaciones Fisicoquímicas Teóricas y Aplicadas (INIFTA), Sucursal 4, Casilla de Correo 16, 1900 La Plata, Argentina \\ Received 12 September 1994; accepted for publication 5 December 1994
}

\begin{abstract}
A description of solid surfaces of interest for electrochemical reactions which is based on the analysis of scanning tunneling microscopy images is presented. Real single-crystal surfaces are better described as weakly disordered surfaces, in contrast to strongly disordered surfaces. Under certain conditions both anisotropic and isotropic strongly disordered surfaces exhibit fractal properties. The rationale of rough surfaces can be attempted by applying the dynamic scaling theory to analyze STM profiles. Examples from regular to irregular solid surfaces are given covering from real single-crystal and faceted metals to columnar structured and branched metal surfaces. Roughness caused by adsorbates and electropolymer coatings are also considered.
\end{abstract}

Keywords: Dendritic and/or fractile surfaces; Surface defects; Surface roughening; Surface structure

\section{Introduction}

A solid surface always exhibits at room temperature some type of disorder. The characterization of surface disorder in surface science and, in particular, in electrochemistry is of outmost importance for the interpretation of experimental data on surface reactions in terms of models derived from statistical mechanics and quantum chemistry [1].

This work presents a systematic description of solid electrode surface disorder in the $\mathrm{nm}$ and $\mu \mathrm{m}$ range which is mainly based upon data derived from scanning tunneling microscopy (STM) [2,3], atomic force microscopy (AFM) [4,5], and scanning electron microscopy complemented with electrochemical data.

* Corresponding author. Fax: +5421 254642 .

\section{Ordered and weakly disordered surfaces}

The use of single-crystal surfaces represents a decisive step forward in understanding electrochemical systems [6-8], although several questions about the actual definition of these surfaces arising from surface science still remain a matter of discussion [9]. In this respect, studies using AFM and STM techniques reveal that single-crystal surfaces used in electrochemistry are considerably distinct from ideally smooth solid single-crystal surfaces $[10,11]$. Perhaps, the C(0001) surface is one of the solid surfaces approaching relatively large defect-free domains. For instance, STM and AFM images for C(0001) surfaces show domains in the $\mu \mathrm{m}$ size range without or with only one or two steps atomic height. The $\mathrm{C}(0001)$ surface exhibits a large stability and, with a few exceptions, an extremely low surface reactivity, 
making it favorable to get atomic resolution even in air. Accordingly, the surface disorder introduced by adsorbates is considerably low. By a simple exfoliation $\mathrm{C}(0001)$ behaves as a clean and atomically flat surface for studying a number of electrochemical reactions of electrocatalytic interest, such as $\mathrm{Pt}, \mathrm{Ag}$, and S electrodeposition by in situ and ex situ STM, combining surface imaging with data resulting from electrochemical and surface analysis techniques $[12,13]$. For these deposits, steps act as active sites for the nucleation and growth of the new phase (Fig. 1). For the growth of $\mathrm{Pt}$ and $\mathrm{Ag}$ electrodeposits from aqueous acid solutions containing dissolved metal ions, the kinetics of the metal phase growth approaches a Volmer-Weber type mechanism [12]. At the early stages of growth, STM images show some crystals $50 \mathrm{~nm}$ in average size which are extremely smooth. Atomic resolution STM images at these domains can be achieved.

On the other hand, S electrodeposits on C(0001) can produce different surface structures depending on the applied potential and the degree of surface coverage by S atoms. STM images of some of these lattices at the monolayer level show the "atomic roughness" introduced by the adsorbed $\mathrm{S}$ atoms (Fig. 2), which can be considered as a chemically induced defect on the C(0001) surface.

The above picture changes considerably for a number of metal single-crystal surfaces usually employed in electrochemical kinetic research work [8]. From our experience, and also from different data reported in the literature $[10,14]$, the surface order in these cases is usually constrained to a surface area in the order of a few $\mathrm{nm}^{2}$. Structural defects such as monoatomic steps, multiple atomic height steps, kinks, corners, atomic clusters, pits, mountain and valley structures can commonly be detected when these surfaces are imaged in the $\mathrm{nm}$ range (Fig. 3). Otherwise, the relatively high surface mobility of metal atoms at the metal electrode/solution interface at room temperature, especially for soft metals [15], favors adsorbate (either anion or organic molecule)-

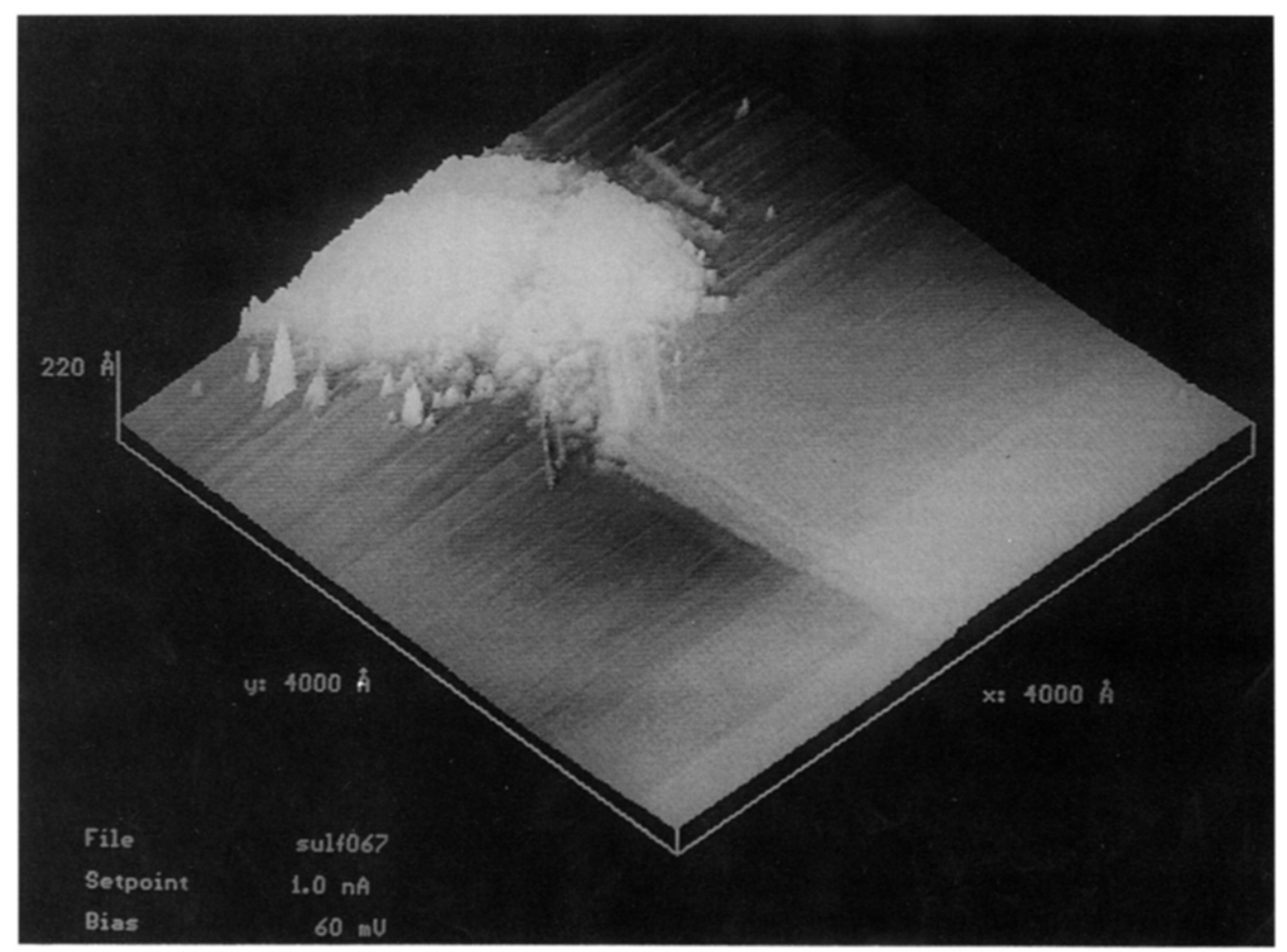

Fig. 1. STM topographic image of a $\mathrm{C}(0001)$ surface with a sulphur agglomerate largely covering a step defect of the substrate. Tunneling current $1 \mathrm{nA}$; bias voltage $60 \mathrm{mV}$. 
induced surface atom rearrangements [14]. Furthermore, reconstructed and unreconstructed surface domains can coexist with structural defects leading to highly heterogeneous surfaces. Thus, it is not surprising that the voltammetric response of these surfaces, can hardly be distinguished from those more disordered surfaces resulting from either the electrochemical faceting of single-crystal metal surfaces prepared in aqueous environment by the application of an adequately selected periodic potential routine [16] or films deposited from metal vapor on a foreign substrate such as mica [17].

Seemingly, the influence of a rather small density of surface defects at the so-called "well-defined surfaces" could explain the discrepancy between the electrochemical behavior of single-crystal metal electrodes in aqueous environment and that of single- crystal surfaces in contact with a gas phase under ultra-high-vacuum (UHV) conditions [9,18,19]. A typical example results from the comparison of the $\mathrm{Pt}-\mathrm{H}$ atom interaction in both systems $[6,20]$. Thus, the degree of surface coverage of $\mathrm{Pt}(111)$ by $\mathrm{H}$ atoms attained under UHV conditions [20] is extremely low and differs considerably from that observed for $\mathrm{H}$ atoms at the $\mathrm{Pt}(111) /$ acid solution electrochemical interface at room temperature [6].

According to the preceding description, it appears that the usually called smooth metal surfaces in electrochemistry could be better described as weakly disordered irregular surfaces [21,22]. Reproducible topographies of this type can be obtained with a preferred crystallographic orientation either with a real surface area almost similar or drastically different from that of the starting material [23]. For this

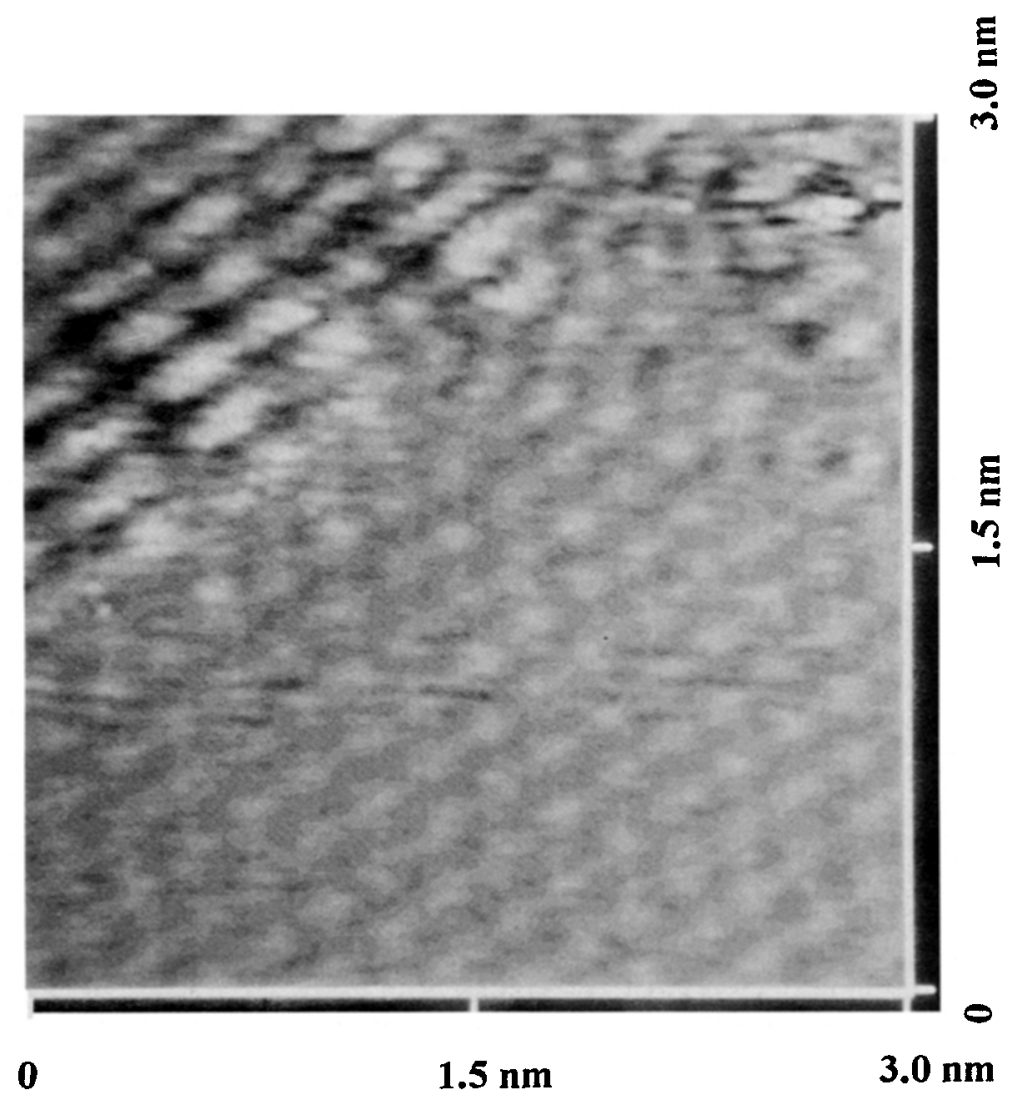

Fig. 2. Atomic resolution constant height STM image (top view) $\left(3 \times 3 \mathrm{~nm}^{2}\right)$ showing a C(0001) domain (lower portion) and a sulphur domain (upper portion). The sulphur submonolayer was produced by electroadsorption from a $10^{-3} \mathrm{Na}_{2} \mathrm{~S}+$ boric acid-sodium borate buffer solution, $\mathrm{pH} 8$, at $298 \mathrm{~K}$. Tunneling current $1 \mathrm{nA}$; bias voltage $2.1 \mathrm{mV}$. 
purpose electrofaceting [16] and electroroughening procedures, which are easily applicable to either a polyfaceted single crystal or a polycrystalline metal [23], have been described in the literature [16,24]. In the case of $\mathrm{Pt}$, each surface topography resulting from these procedures leads to a specific $\mathrm{H}$-atom electroadsorption voltammogram in a particular acid solution [23].

\section{Strongly disordered systems}

Continuous strongly disordered metal surfaces can be prepared by physical and electrochemical methods leading to the development of either anisotropic or isotropic strongly disordered surfaces [22,24,25]. This type of surface is important for producing high surface area metal electrodes, for establishing the concept of roughness, structural and kinetic models for surface roughening, and for providing more reliable criteria for the evaluation of solid electrocatalyst active surface area.

\subsection{Anisotropic surface disorder}

An anisotropic strongly disordered topography is closely approached by a columnar-structured metal surface produced at growth rates in the $0.1-100 \mathrm{~nm}$ $\mathrm{s}^{-1}$ range under a surface reaction control. This is the case of metal deposits made on a smooth substrate by either vapor deposition [17] or metal ion electrodeposition from dissolved metal ions in solution [26] or hydrous metal oxide layer electroreduction in aqueous solution [23,24].

A number of columnar-structured metal surfaces exhibit a self-affine fractal surface and a non-fractal

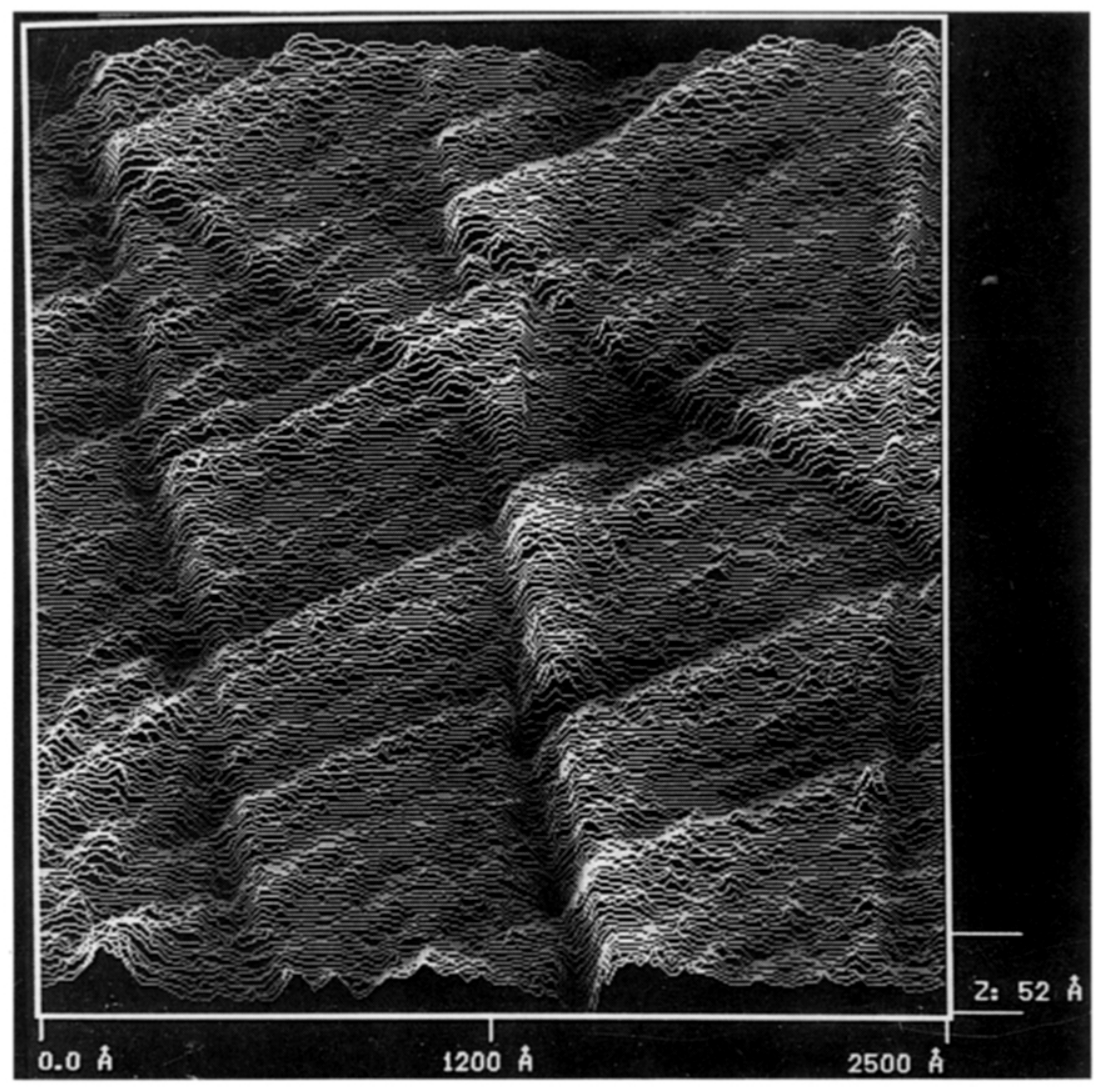

Fig. 3. STM image (scan lines) of a Pt single crystal which was prepared from a polyfaceted Pt single crystal by cutting normally to the [111] direction and flame annealed for $5 \mathrm{~min}$ at $\sim 1470 \mathrm{~K}$. Tunneling current $3 \mathrm{nA}$; bias voltage $240 \mathrm{mV}$. 
mass. In this case, the degree of surface disorder can be quantitatively approached by means of the dynamic scaling theory [27]. Accordingly, $\xi$, the interface width, scales as

$\xi(L, h) \propto L^{\alpha} f(x)$,

where $L$ is the substrate length, $\alpha$ is the characteristic surface roughness exponent, $f(x)=\langle h\rangle / L^{\alpha / \beta}$, $f(x)=$ const for $\mathrm{x} \rightarrow \infty$, and $f(x)=x^{\beta}$ for $x \rightarrow 0 . \xi$ is defined by

$\xi(L)=\left((1 / N) \sum\left[h\left(x_{1}\right)-\langle h\rangle\right]^{2}\right)^{1 / 2}$,

where $h\left(x_{i}\right)$ is the deposit height measured along the $x$-direction at the position $x_{i}$, and $\langle h\rangle$ is the average height of the sample formed by $N$ points. After a certain critical time or thickness has been reached, Eq. (1) becomes

$\xi(L) \propto L^{\alpha}$.
The exponent $\alpha$, the static scaling exponent, is a relevant quantity in the theory because it is a measure of the surface disorder and is related to $D_{\mathrm{s}}$, the local fractal dimension of the self-affine surface, through

$D_{\mathrm{s}}=d-\alpha$,

where $d$ is the deposit growth dimension. For the range $0<\alpha<1, \alpha$ increases as the degree of surface disorder decreases.

Values of $\alpha$ for solid surfaces can be derived from different methods based on the analysis of STM or AFM images [28-30]. Thus, a $\log \xi(L)$ versus $\log L$ plot of Eq. (3) for both weakly disordered surfaces such as single-crystal faceted metal surfaces and vapor-deposited films grown at high substrate temperature $\left(T_{\mathrm{s}}=630 \mathrm{~K}\right)$ (Fig. 4$)$ results in a single linear regime with $\alpha \approx 1$, whereas for those surfaces grown under a surface reaction control in

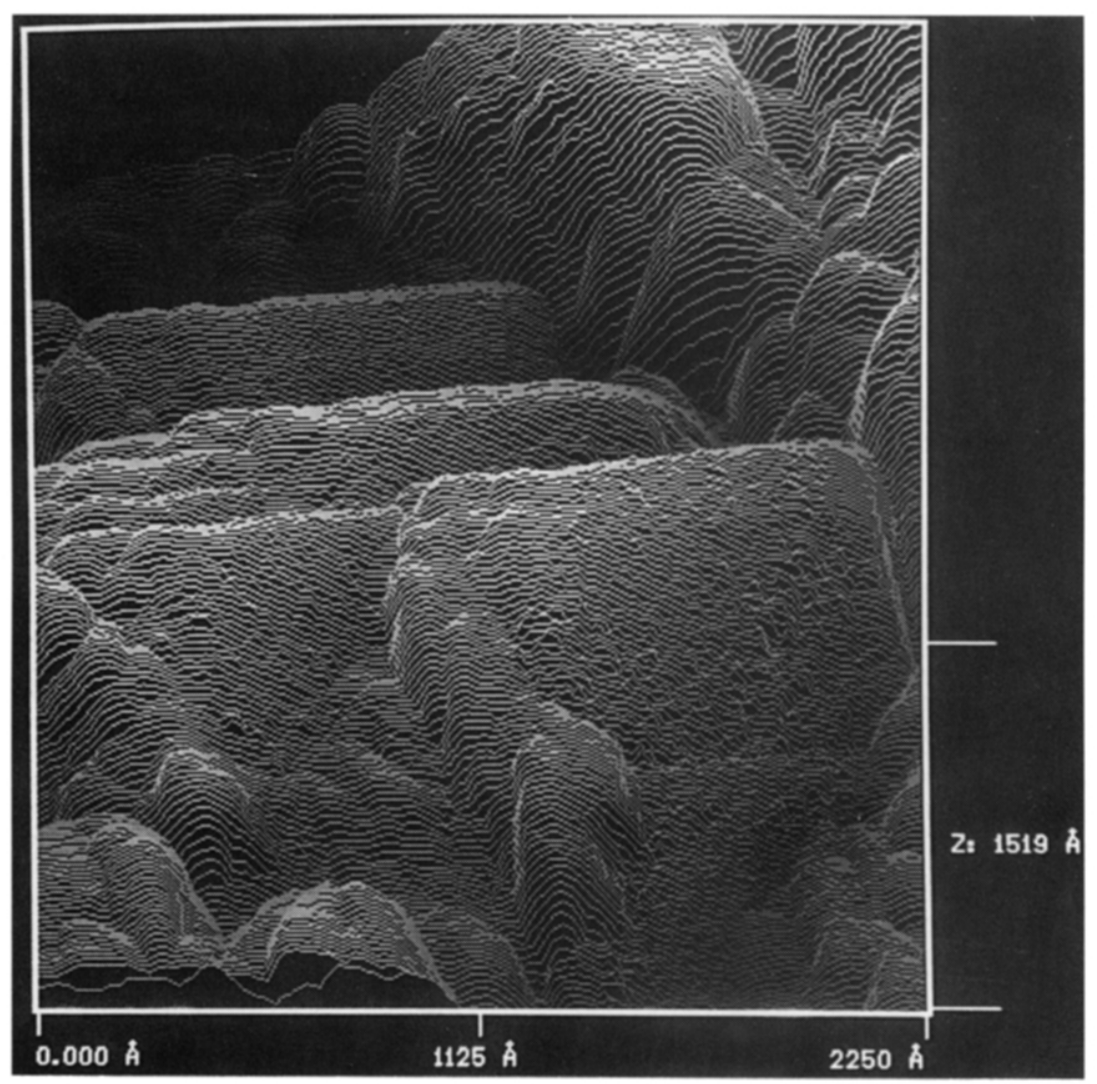

Fig. 4. STM image (scan lines) of vapor-deposited gold on glass $\left(T_{\mathrm{s}}=600 \mathrm{~K}\right)$. Tunneling current $3 \mathrm{nA}$; bias voltage $40 \mathrm{mV}$. 
the absence of surface diffusion, such as the surface of conducting polymer coatings (Fig. 5) it results $\alpha \approx 0.35$. This figure is close to the value of $\alpha$ predicted by the Kardar, Parisi and Zhang surface growth model (KPZ) [31]. The motion equation for this model is

$$
(\partial h / \partial t)=\nu \nabla^{2} h+(\lambda / 2)(\nabla h)^{2}+\eta,
$$

where $h$ is the height of the surface at time $t$ above the specified substrate site, the $\lambda$ term determines the average growth velocity which may depend on the tilt of the surface, the $\nu$ term has a smoothing effect on the surface, and $\eta$ denotes the white noise due to fluctuations in the growth rate which makes the surface rough.

On the other hand, a $\log \xi(L)$ versus $\log L$ plot for vapor-deposited gold surfaces grown at a low temperature $\left(T_{\mathrm{s}}=298 \mathrm{~K}\right)$ (Fig. 6) exhibits two linear regimes leading to $\alpha$ (I) and $\alpha$ (II) values, depending whether $L$ is greater or smaller than $d_{\mathrm{s}}$, the average columnar size. Accordingly, as shown in Table 1 for different systems, for $L\left\langle d_{\mathrm{s}},\langle\alpha(\mathrm{I})\rangle \approx 0.9\right.$, whereas for $L>d_{\mathrm{s}},\langle\alpha(\mathrm{II})\rangle \approx 0.4$, in agreement with the $\mathrm{KPZ}$ model.

The dynamic scaling analysis of surface profiles

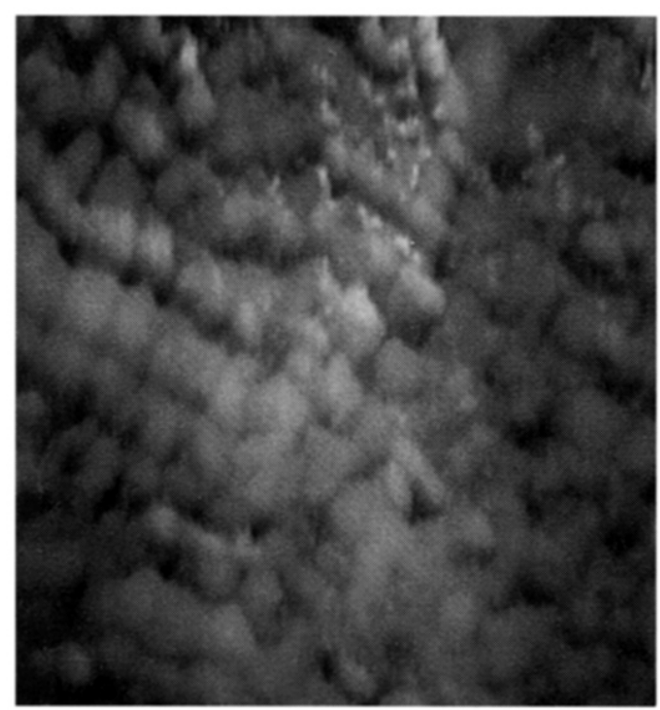

Fig. 5. STM image (top view) $280 \times 280 \mathrm{~nm}^{2}$ of a poly-o-toluidine coating on a gold single-crystal substrate. Electropolymerization was run at a constant potential $(E=0.35 \mathrm{~V}$ versus a $\mathrm{Hg} / \mathrm{Hg}_{2} \mathrm{SO}_{4} / 0.5 \mathrm{M} \mathrm{H} \mathrm{H}_{2} \mathrm{SO}_{4}$ reference electrode) in a $0.5 \mathrm{M}$ $\mathrm{H}_{2} \mathrm{SO}_{4}+0.65 \mathrm{M}$ o-toluidine aqueous solution at $298 \mathrm{~K}$. of gold films produced by electroreducing hydrous gold oxide layers in acid solution at $298 \mathrm{~K}$ [32] also fit Eq. (3) yielding for $L\left\langle d_{\mathrm{s}},\langle\alpha(\mathrm{I})\rangle \approx 0.9\right.$, and for $L>d_{\mathrm{s}},\langle\alpha(\mathrm{II})\rangle \approx 0.5$ [33]. This value of $\alpha(\mathrm{II})$, however, cannot be straightforwardly explained by the growth models available at present. Nevertheless, the columnar size itself imposes an inner cutoff to the self-affine behavior of the surface.

The properties of the above described irregular metal surfaces immersed in a conducting solution as well as those of any other metal depend on the substrate-ion and substrate-solvent interactions which are obviously specific to each electrochemical system. Thus, there is a topography change in a vapor-deposited gold film grown at $T_{\mathrm{s}}=298 \mathrm{~K}$ when it is immersed in aqueous $0.5 \mathrm{M} \mathrm{H}_{2} \mathrm{SO}_{4}+0.5 \mathrm{M} \mathrm{NaCl}$ solution at $323 \mathrm{~K}$, which reflects in the gradual change in the $\log \xi(L)$ versus $\log L$ plot approaching a single linear regime with $\alpha \approx 0.9$. In this case the gold surface becomes increasingly ordered and the topography change can be interpreted through the proper surface mobility of gold atoms. In fact, the surface mobility of metal atoms can be enhanced not only by increasing $T_{\mathrm{s}}$ but also by the presence of adsorbable species in the environment $[34,35]$. Therefore, gold surface roughness relaxation is induced by chloride ions present in the solution. The adsorption of these anions on gold increases the value of the average surface diffusion coefficient of gold atoms from $D \approx 10^{-14} \mathrm{~cm}^{2} \mathrm{~s}^{-1}$ for $c_{\mathrm{NaCl}}=0$ to $D=10^{-11} \mathrm{~cm}^{2} \mathrm{~s}^{-1}$ for $c_{\mathrm{NaCl}}=0.05 \mathrm{M}$ at $298 \mathrm{~K}$ $[34,35]$.

The change from the columnar (self-affine) surface to the faceted surface induced by changing $T_{\mathrm{s}}$ can be interpreted by using Villain's model [36]. The corresponding surface height $(h)$ growth rate equation is

$$
\begin{gathered}
(\partial h / \partial t)=\nu \nabla^{2} h+\lambda(\nabla h)^{2}+K \nabla^{2}\left(\nabla^{2} h\right) \\
+\sigma \nabla^{2}(\nabla h)^{2}+F+\eta,
\end{gathered}
$$

where $F$ is the net deposition flux, and $\eta$ is the $\delta$-function correlated noise. The $\nu$ and $\lambda$-containing terms appear when desorption is operative [37]. The $\nu$-containing term is related to the surface tension [38], and the $\lambda$-containing term to the dependence of growth rate on $\lambda$, the local tilt [39], whereas the $K$ and $\sigma$-containing terms are related to surface diffu- 


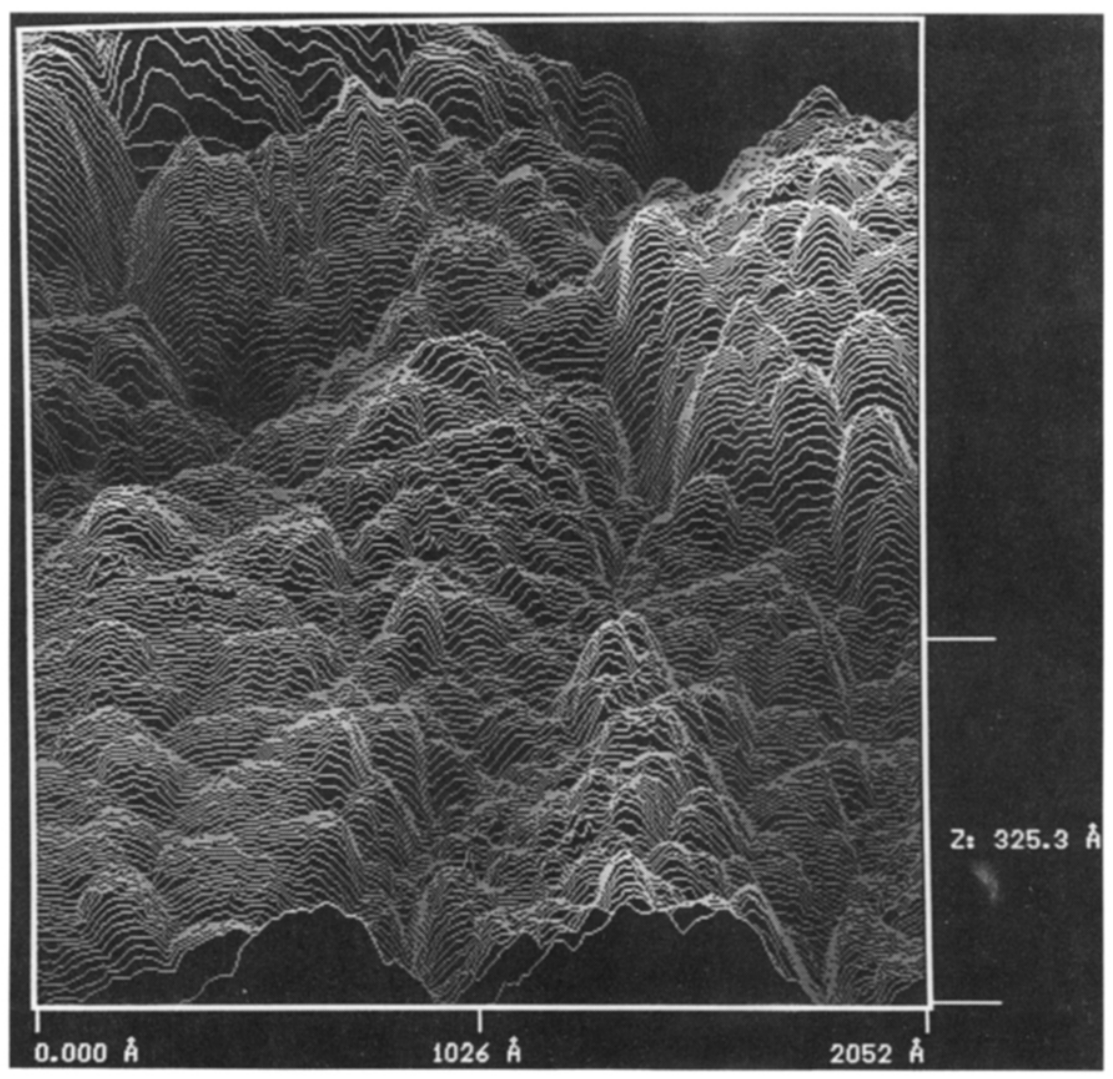

Fig. 6. STM image (scan lines) of vapor-deposited gold on glass $\left(T_{\mathrm{s}}=298 \mathrm{~K}\right)$. Tunneling current $3 \mathrm{nA}$; bias voltage $40 \mathrm{mV}$.

sion [36]. Note that for $K=0$ and $\sigma=0$ Eq. (6) leads to Eq. (5).

When surface diffusion becomes irrelevant Eq.
(6) leads to $\alpha \approx 0.4$ as predicted by the $\mathrm{KPZ}$ model. On the other hand, when surface diffusion is operative, $K$ and $\sigma$ are non-zero, crossover phenomena

Table 1

Average surface roughness exponents derived from the dynamic scaling theory for various experimental systems

\begin{tabular}{|c|c|c|c|c|c|c|}
\hline Surface & $\begin{array}{l}T_{\mathrm{s}} \\
(\mathrm{K})\end{array}$ & Environment & $\begin{array}{l}t_{\mathrm{a}} \\
(\mathrm{s})\end{array}$ & $\begin{array}{l}T_{\mathrm{e}} \\
(\mathrm{K})\end{array}$ & $\langle\alpha(\mathrm{I})\rangle$ & $\langle\alpha(\mathrm{II})\rangle$ \\
\hline $\mathrm{Au}(\mathrm{v})$ & 298 & $P<10^{-4}$ Torr & - & - & $0.90 \pm 0.07$ & $0.40 \pm 0.05$ \\
\hline $\mathrm{Au}(\mathrm{v})$ & 298 & $\mathrm{H}_{2} \mathrm{SO}_{4}$ & $10^{4}$ & 323 & $0.90 \pm 0.07$ & $0.43 \pm 0.04$ \\
\hline$A u(v)$ & 298 & $\mathrm{H}_{2} \mathrm{SO}_{4}+0.01 \mathrm{M} \mathrm{NaCl}$ & $10^{4}$ & 323 & $0.90 \pm 0.07$ & $0.55 \pm 0.07$ \\
\hline$A u(v)$ & 298 & $\mathrm{H}_{2} \mathrm{SO}_{4}+0.5 \mathrm{M} \mathrm{NaCl}$ & $10^{4}$ & 323 & $0.90 \pm 0.07$ & $0.56 \pm 0.06$ \\
\hline $\mathrm{Au}(\mathrm{v})$ & 673 & $P<10^{-4}$ Torr & - & - & $0.90 \pm 0.07$ & - \\
\hline $\mathrm{Au}(\mathrm{e})$ & 298 & $\mathrm{H}_{2} \mathrm{SO}_{4}$ & - & 298 & $0.90 \pm 0.07$ & $0.49 \pm 0.07$ \\
\hline $\mathrm{Au}(\mathrm{e})$ & 298 & $\mathrm{H}_{2} \mathrm{SO}_{4}$ & $10^{4}$ & 323 & $0.80 \pm 0.05$ & - \\
\hline $\mathrm{Au}(\mathrm{sc})$ & - & - & - & - & $0.99 \pm 0.03$ & - \\
\hline $\mathrm{Pt}(\mathrm{e})$ & 298 & $\mathrm{H}_{2} \mathrm{SO}_{4}$ & - & - & - & $0.5 \pm 0.1$ \\
\hline $\operatorname{Pt}(\mathrm{e})$ & 298 & $\mathrm{H}_{2} \mathrm{SO}_{4}$ & $10^{4}$ & 323 & - & $0.5 \pm 0.1$ \\
\hline $\mathrm{Au}(\mathrm{sc}) /$ poly-(o-toluidine $)$ & 298 & $\mathrm{H}_{2} \mathrm{SO}_{4}$ & - & 298 & $0.85 \pm 0.05$ & $0.33 \pm 0.05$ \\
\hline
\end{tabular}

$\mathrm{v}$, vapor; e, electrodeposit; sc, single crystal; $P$, pressure; $T_{\mathrm{s}}$, substrate temperature; $T_{\mathrm{e}}$, environment temperature; $t_{\mathrm{a}}$, ageing time. 
may occur as observed for vapor-deposited gold films for $T_{\mathrm{s}}=298 \mathrm{~K}$. On the high temperature limit $\left(T_{\mathrm{s}}=673 \mathrm{~K}\right)$, models in which particles are randomly deposited onto a substrate and subsequently relax to kink sites maximizing the number of saturated bonds, can be used to describe the surface topography $[40,43]$. Thus, a continuum model incorporating surface relaxation $[42,43]$ developed for the molecular beam epitaxial growth at high temperature leads to $\alpha=2 / 3$ rather than $\alpha \approx 0.9$ in 3D growth, as obtained from the experimental data. On the other hand, in a $3 D$ space the value $\alpha \approx 1$ is predicted by the 3D growth model presented in Ref. [36], whereas the model presented in Ref. [37] leads to intermediate values, and in generic cases the system shows either a completely smooth surface $(\alpha=0)$ or $\alpha=1$ [42-44].

The value $\alpha \approx 0.9$ means that surface restructuring may be too strong to remove all surface irregularities yielding large facets with a smooth texture. However, for high $T_{\mathrm{s}}$ and $t \rightarrow \infty$, the growth of faceted crystals could lead to a completely smooth surface. Thus, the value $\alpha \approx 0.9$ for high $T_{\mathrm{s}}$ can be assigned to a transient regime of the growing sur- face, and then a crossover to the Edwards-Wilkinson (sedimentation model) scaling [44] $(\alpha=0)$ could be expected.

Self-affine fractal metal electrodes may exhibit anomalous adsorption behavior according to the size of the adsorbate. This can be demonstrated by scaling the electrode surface with adsorbates (yardsticks) of different cross-sections. Thus, for aged electrodeposited columnar gold electrodes $(\alpha \approx 0.8)$ immersed in aqueous $0.5 \mathrm{M} \mathrm{H} \mathrm{H}_{2} \mathrm{SO}_{4}+0.1 \mathrm{M} 1,10-$ phenantroline at $298 \mathrm{~K}$, the degree of surface coverage by this molecule decreases sharply as the electrode real surface area increases, whereas it remains unchanged when smaller adsorbates such as glucose or $\mathrm{CO}_{2}$ [45] are used. Results from 1,10-phenantroline adsorption reflect the influence of excluded volume regions in the proper electrode structure due to small and deep intercolumnar voids with a diameter in the order of the adsorbate cross-section. In this case void walls become unaccessible sites for the 1,10-phenantroline adsorption.

On the other hand, the development of irregular surfaces may exhibit a transition which occurs during the new phase growth and which can be exempli-

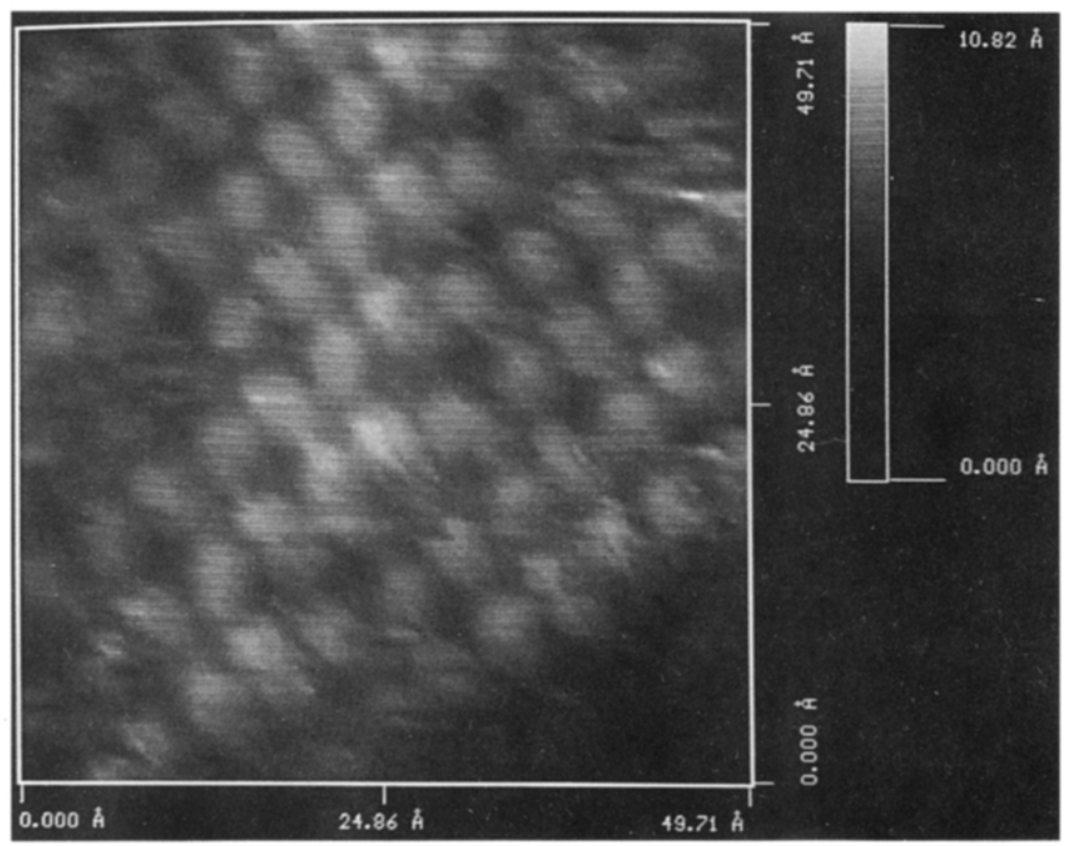

Fig. 7. STM image in the topographic mode (top view) of a polyaniline domain on vapor-deposited gold on glass $\left(T_{\mathrm{s}}=600 \mathrm{~K}\right.$ ). The average size of the ordered structure is $0.50 \times 0.65 \mathrm{~nm}^{2}$. Tunneling current $7 \mathrm{nA}$; bias voltage $20 \mathrm{mV}$. 
fied by the electroformation of a conducting polymer coating on a smooth metal substrate. For polyaniline conducting films grown from aqueous $0.5 \mathrm{M} \mathrm{H}_{2} \mathrm{SO}_{4}$ $+0.1 \mathrm{M}$ aniline solution up to $2-3 \mathrm{~nm}$ thick, a structure made of ordered parallel monomer chains forming a rectangular array of about $0.50 \times 0.65$ $\mathrm{nm}^{2}$ is observed (Fig. 7) [46]. In this case for image size, typically $20 \mathrm{~nm}^{2}$ domains, the corrugation of the deposit is in the order of $0.1 \mathrm{~nm}$ (Fig. 7), a figure which is close to that reported from STM data for the corrugation of adsorbed benzene on different metal substrates [47]. Thus, the electropolymer coated surface can be described as atomically smooth because in this case roughness is largely due to the proper charge density periodicity of polyaniline monomers.

When the amount of electrodeposit is increased leading to a film of $10 \mathrm{~nm}$ in average thickness, monomer chains are replaced by a fiber-like struc- ture about $2.5 \mathrm{~nm}$ wide. Finally, when the film thickness is in the $\mu \mathrm{m}$ range, globular structures form disordered domains leading to a rough surface (Fig. 5) with $\alpha=0.35$, a figure close to the predictions of both the Eden [27] and KPZ [31] growth model.

\subsection{Isotropic disordered systems}

Ideally isotropic strongly disordered surfaces are approached by branched metal electrodeposits comprising dense radial, dendritic and diffusion-limited aggregation (DLA-like) deposits [48]. This type of electrodeposit can be obtained at growth rates higher than $100 \mathrm{~nm} \mathrm{~s}^{-1}$, typical values are $(1-3) \times 10^{-3}$ $\mathrm{cm} \mathrm{s}^{-1}$, under mass transport control.

DLA-like electrodeposits exhibit surface fractal and mass fractal properties which are characterized by $D_{\mathrm{s}}$, the surface fractal dimension, and $D_{\mathrm{M}}$, the

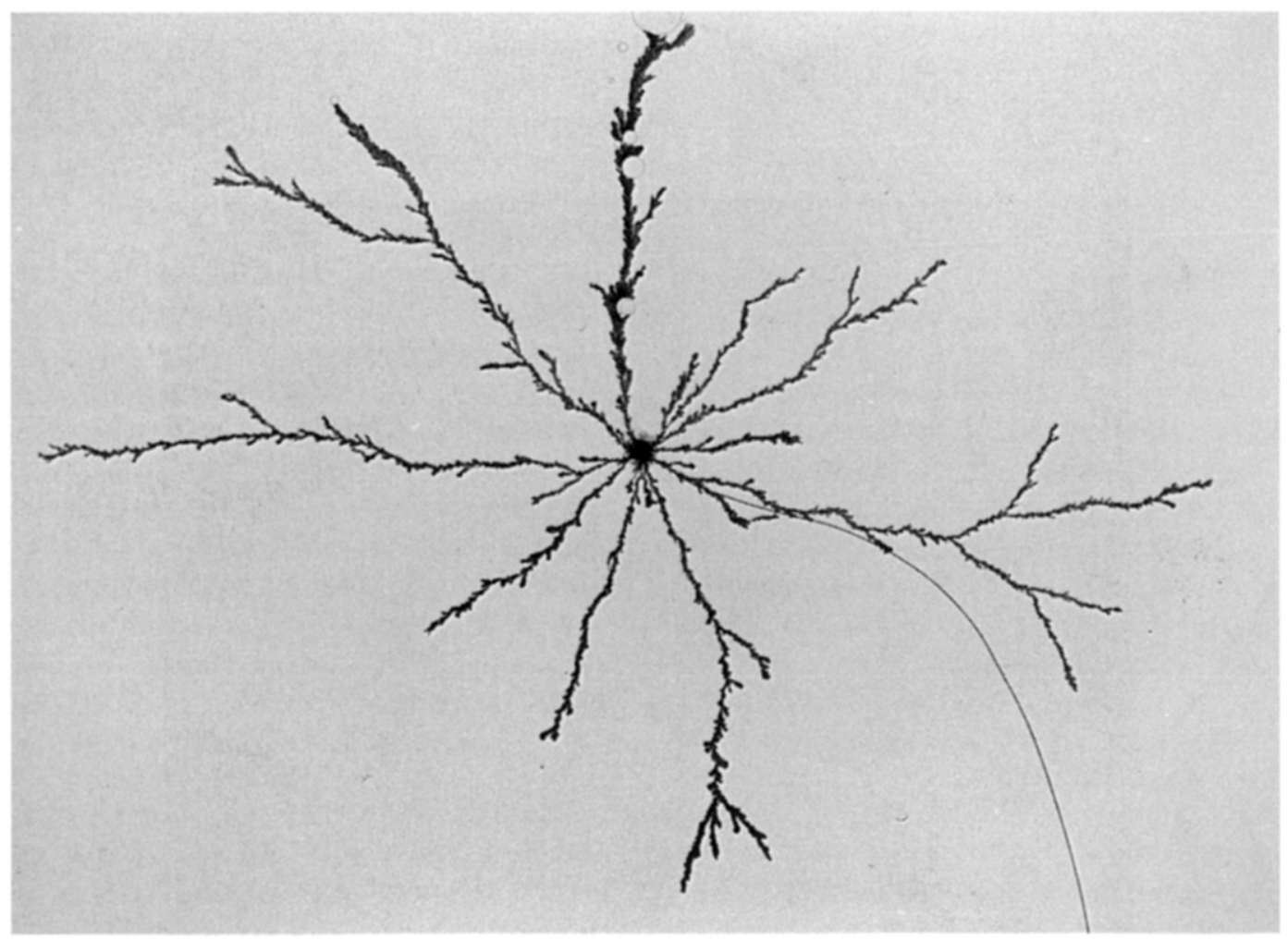

Fig. 8. A DLA-like pattern of a copper electrodeposit produced at the center of a quasi-2D cylindrical cell $15 \mathrm{~cm}$ in outer diameter and 0.1 $\mathrm{mm}$ in height, from a $0.4 \mathrm{M} \mathrm{CuSO}_{4}$ aqueous solution at $298 \mathrm{~K}$. A $15 \mathrm{~V}$ constant potential was applied between the anode and the cathode of the cell. 
mass fractal dimension, respectively. For a 3D-grown object $D_{\mathrm{s}}=D_{\mathrm{M}} \approx 2.5$, whereas $D_{\mathrm{s}}=D_{\mathrm{M}} \approx 1.7$ in a 2D space. A typical DLA-like pattern of a copper electrodeposit grown in a 2D electrochemical cell is shown in Fig. 8.

Dendritic electrodeposits can exhibit values of $D_{\mathrm{s}}$ and $D_{\mathrm{M}}$ close to or lower than those corresponding to theoretical DLA patterns. Otherwise, dense radial electrodeposits are only surface fractals with either $D_{\mathrm{s}} \approx 2.5$ for a 3D-grown object, or $D_{\mathrm{s}} \approx 1.7$ for a 2D-grown object. In both cases it results in $D_{\mathrm{M}} \approx d$, ie. an Euclidean mass. A typical 2D-non-fractal mass silver electrodeposit can be obtained at the early stage of growth as seen in Fig. 9. Subsequently, various transitions in the morphology of the deposit can be distinguished.

Pattern selection depends on the relative contribution of mass transport processes (migration, diffusion and convection) $[49,50]$. When convection and migration contribute to the growth process dense radial patterns are usually developed, whereas when they become negligible DLA-like patterns can be observed [51,52]. Often the object macroscopic shape, and accordingly the radial growth rate, depend mainly on the migration and surface reaction contributions, whereas the object microscopic pattern where most of the deposit mass is accumulated, can be modeled by diffusion leading to $D_{\mathrm{M}}$ values compatible with DLA models.

\section{Acknowledgements}

This research project was financially supported by the Consejo Nacional de Investigaciones Científicas

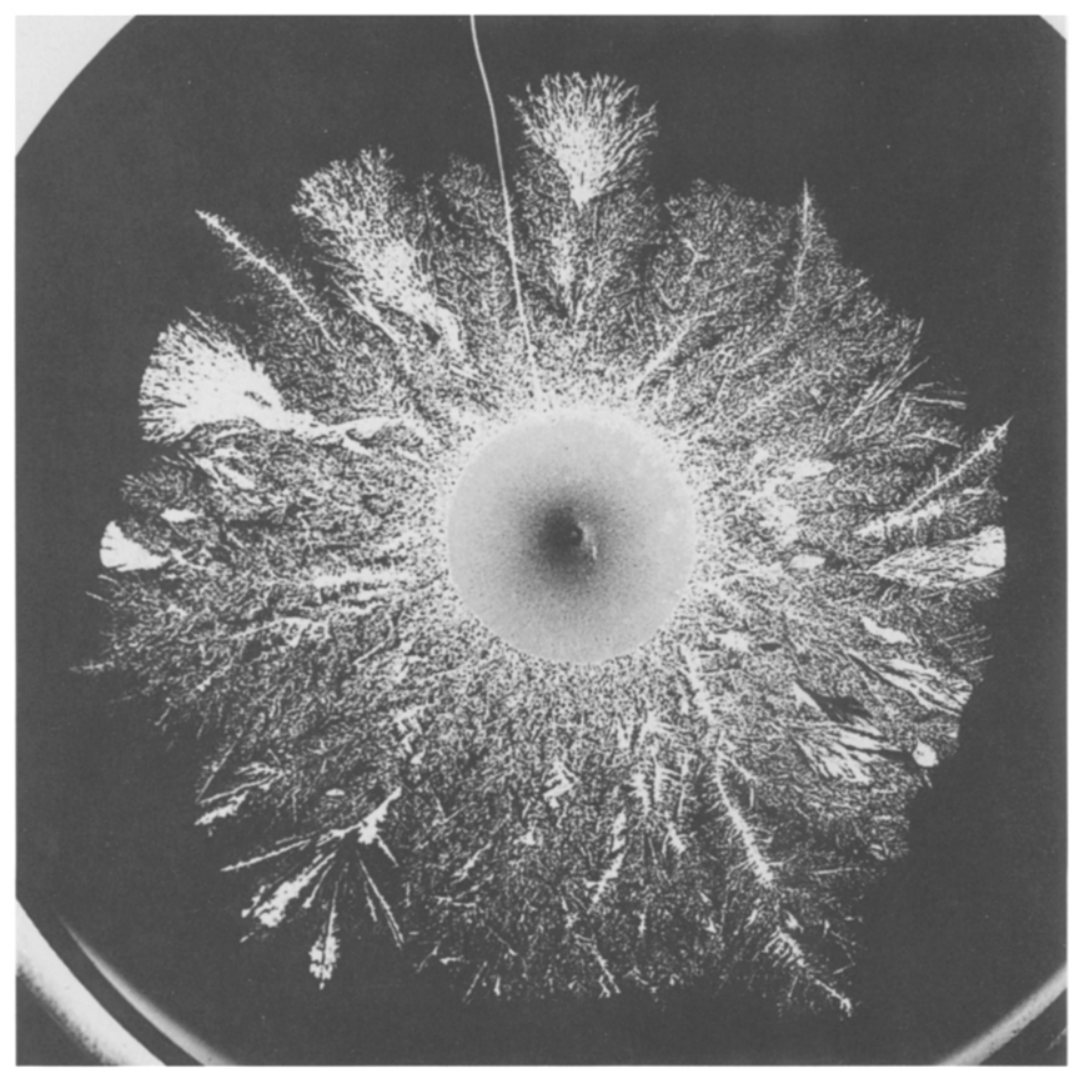

Fig. 9. Silver electrodeposit approaching a dense radial pattern during the initial electrodeposition stage. Afterwards a transition to more complex branched structures can be observed. The electrodeposit was formed from a $0.024 \mathrm{M} \mathrm{Ag} \mathrm{AO}_{4}+0.01 \mathrm{M} \mathrm{H}_{2} \mathrm{SO}_{4}+0.5 \mathrm{M} \mathrm{Na} \mathrm{SO}_{4}$ aqueous solution at $298 \mathrm{~K}$ using a quasi-2D cylindrical electrochemical cell by keeping a constant cathodic overpotential (0.042 $\mathrm{V})$ electrode. 
y Técnicas and the Comisión de Investigaciones Científicas de la Provincia de Buenos Aires.

\section{References}

[1] J. Lapujoulade, Surf. Sci. Rep. 20 (1994) 191.

[2] G. Binnig, H. Röhrer and E. Weibel, Phys. Rev. Lett. 50 (1983) 120.

[3] H. Röhrer, Surf. Sci. 299/300 (1994) 956.

[4] G. Binnig, C.F. Quate and C. Gerber, Phys. Rev. Lett. 56 (1986) 930.

[5] F. Lin and D.J. Meier, Langmuir 10 (1994) 1660.

[6] J. Clavilier and J.P. Chauvineau, J. Electroanal. Chem. 100 (1978) 461

[7] S. Motoo and N. Furuya, J. Electroanal. Chem. 172 (1978) 339 ; N. Furuya and S. Koide, Surf. Sci. 220 (1989) 18.

[8] D.M. Kolb, in: Advances in Electrochemistry and Electrochemical Engineering, Vol. 11, Eds. H. Gerischer and C.W. Tobias (Wiley-Interscience, New York, 1978) p. 125; R.R. Adzic, in: Advances in Electrochemistry and Electrochemical Engineering, Vol. 13, Eds. H. Gerischer and C.W. Tobias (Wiley-Interscience, New York, 1984) p. 159.

[9] T.E. Furtak, Surf. Sci. 299/300 (1994) 945.

[10] N. Kimizuka, T. Abe and K. Itaya, Denki Kagaku 81 (1993) 796.

[11] Z. Gao, A. Hamelin and M. Weaver, Phys. Rev. B 46 (1992) 1096.

[i2] L. Vázquez, A. Hernández Creus, P. Carro, P. Ocón, P. Herrasti, C. Palacio, J.M. Vara, R.C. Salvarezza and A.J. Arvia, J. Phys. Chem. 96 (1992) 10454.

[13] F. Ogletree and M. Salmeron, Prog. Solid State Chem. 20 (1990) 235

[14] D.J. Trevor, C.E. Chidsey and D.N. Loiaccono, Phys. Rev. Lett. 62 (1980) 929

[15] M.E. Martins, R.C. Salvarezza and A.J. Arvia, J. Electrochem. Soc., submitted.

[16] A.J. Arvia, J.C. Canullo, E. Custidiano, C.L. Perdriel and W.E. Triaca, Electrochim. Acta 31 (1986) 1359.

[17] J.L. Zubimendi, M.E. Vela, R.C. Salvarezza, L. Vázquez, J.M. Vara and A.J. Arvia, Phys. Rev. 50 (1994) 1367.

[18] C. Lamy, J.M. Leger, J. Clavilier and R. Parsons, J. Electroanal. Chem. 150 (1983) 71.

[19] E.P. Leiva, E. Santos, R.M. Cerviño, M.C. Giordano and A.J. Arvia, Electrochim. Acta 30 (1985) 1111.

[20] B.J.J. Koeleman, S.T. de Zwart, A.L. Boers, B. Poelsema and L.K. Verhey, Nucl. Instrum. Methods Phys. Res. 218 (1983) 225

[21] H.P. Bonzel, in: Surface Physics of Materials, Ed. J.M. Blakely (Academic Press, New York, 1975) p. 280.

[22] R.C. Salvarezza and A.J. Arvia, in: Modern Aspects of Electrochemistry, Vol. 28, Eds. J.O'M. Bockris and B.E. Conway, in press.
[23] A. Visintin, J.C. Canullo, W.E. Triaca and A.J. Arvia, J. Electroanal. Chem. 239 (1988) 67.

[24] A.J. Arvia, R.C. Salvarezza and W.E. Triaca, Electrochim. Acta 34 (1989) 1057.

[25] A.J. Arvia and R.C. Salvarezza, Electrochim. Acta, in press.

[26] A. Hernández Creus, P. Carro, S. González, R.C. Salvarezza and A.J. Arvia, J. Electrochem. Soc. 139 (1992) 1064.

[27] F. Family, Physica A, 168 (1990) 561, and references therein.

[28] R.C. Salvarezza, L. Vázquez, P. Herrasti, P. Ocón, J.M. Vara and A.J. Arvia, Europhys. Lett. 20 (1992) 727.

[29] L. Vázquez, R.C. Salvarezza, P. Herrasti, P. Ocón, J.M. Vara and A.J. Arvia, Appl. Surf. Sci. $70 / 71$ (1993) 413.

[30] P. Herrasti, P. Ocón, L. Vázquez, R.C. Salvarezza, J.M. Vara and A.J. Arvia, Phys. Rev. A 45 (1990) 7440.

[31] M. Kardar, G. Parisi and Y.C. Zhang, Phys. Rev. Lett. 56 (1986) 889.

[32] A.C. Chialvo, W.E. Triaca and A.J. Arvia, J. Electroanal. Chem. 146 (1983) 93.

[33] L. Vázquez, R.C. Salvarezza, P. Ocón, P. Herrasti, J.M. Vara and A.J. Arvia, Phys. Rev. E 49 (1994) 1507.

[34] C. Alonso, R.C. Salvarezza, J.M. Vara and A.J. Arvia, Electrochim. Acta 35 (1990) 1331.

[35] M.P. Garcia, M.M. Gómez, R.C. Salvarezza and A.J. Arvia, J. Electroanal. Chem. 347 (1993) 237.

[36] J. Villain, J. Phys. I 1 (1991) 19.

[37] D.D. Vvdensky, A. Zangwill, C.N. Luse and M.R. Wilby, Phys. Rev. E 48 (1993) 852.

[38] F. Family, in: Fractals in the Natural and Applied Sciences, Ed. M.M. Novak (North-Holland, Amsterdam, 1994) p. 1

[39] Z.W. Amar and F. Family, Phys. Rev. E 47 (1993) 1595.

[40] Z.W. Lai and S. Das Sarma, Phys. Rev. Lett. 66 (1991) 2348.

[41] S. Das Sarma and P. Tamborenea, Phys. Rev. Lett. 66 (1991) 325 .

[42] D. Wolf and J. Villain, Europhys. Lett. 13 (1990) 389.

[43] M. Schroeder, M. Siergert, D.E. Wolf, J.D. Shore and M. Pliechke, Europhys. Lett. 24 (1993) 563.

[44] S.F. Edwards and D.R. Wilkinson, Proc. R. Soc. London A 381 (1982) 17.

[45] M.M. Gómez, M.P. García, J. San Fabián, L. Vázquez, R.C. Salvarezza and A.J. Arvia, submitted.

[46] J.L. Zubimendi, M.E. Vela, R.C. Salvarezza and A.J. Arvia, in preparation.

[47] P. Sautet and M.L. Bocquet, Surf. Sci. 304 (1994) L445.

[48] M. Matsushita, in: The Fractal Approach to the Heterogeneous Chemistry, Ed. D. Avnir (Wiley, New York, 1989) p. 161.

[49] A. Hernández Creus, A.E. Bolzan, P. Carro, S. González, R.C. Salvarezza, S.L. Marchiano and A.J. Arvia, J. Electroanal. Chem. 336 (1992) 85 .

[50] A. Hernández Creus, P. Carro, S. González, R.C. Salvarezza and A.J. Arvia, J. Electrochem. Soc. 139 (1992) 1064.

[51] A. Hernández Creus, P. Carro, S. González, S.L. Marchiano, R.C. Salvarezza and A.J. Arvia, Fractals A 41 (1994) 192.

[52] P.L. Schilardi, S.L. Marchiano, R.C. Salvarezza and A.J. Arvia, Chaos, Solitons and Fractals, in press. 